\title{
ON ADELIC CHERN FORMS AND THE BOTT RESIDUE FORMULA
}

\author{
AMNON YEKUTIELI \\ Department of Theoretical Mathematics, The Weizmann Institute of Science \\ Rehovot 76100, Israel \\ e-mail: amnon@wisdom.weizmann.ac.il
}

0. Introduction. The Bott Residue Formula gained renewed attention recently due to its use in enumerative algebraic geometry (cf. [ES], [Ko]). If $X$ is a smooth projective variety over a field $k$ of characteristic 0 , then Bott's formula makes sense purely algebraically, with the Chern classes taken in the algebraic De Rham cohomology $\mathrm{H}_{\mathrm{DR}}(X / k)$. In this paper we survey an algebraic proof of the formula using Beilinson adeles, which was discovered by R. Hübl and the author (see $[\mathrm{HY}]$ ).

Suppose $v \in \Gamma\left(X, \mathcal{T}_{X}\right)$ is a global vector field with isolated, simple, $k$-rational zeroes (see Remark 3.2 for generalizations). Let $\mathcal{E}_{1}, \ldots, \mathcal{E}_{m}$ be locally free $\mathcal{O}_{X}$-modules. Suppose $\Lambda_{i}$ is an action of $v$ on $\mathcal{E}_{i}$, i.e. a differential operator $\Lambda_{i}: \mathcal{E}_{i} \rightarrow \mathcal{E}_{i}$ satisfying $\Lambda_{i}(a e)=$ $v(a) e+a \Lambda_{i}(e)$ for local sections $a \in \mathcal{O}_{X}, e \in \mathcal{E}_{i}$. Suppose $Q\left(t_{i, j}\right)$ is a homogeneous polynomial of degree $n=\operatorname{dim} X$ in the variables $t_{i, j}\left(i=1, \ldots, m ; j=1, \ldots, r_{i} ; r_{i}:=\right.$ rank $\mathcal{E}_{i}$ ) which have degrees $\operatorname{deg} t_{i, j}=j$. For a zero $z$ of $v$ let us denote by $\left.\Lambda_{i}\right|_{z}$ the restriction of $\Lambda_{i}$ to $\left.\mathcal{E}_{i}\right|_{z}:=\mathcal{E}_{i} \otimes k(z)$, which is a $k$-linear endomorphism. Also let us denote by ad $\left.v\right|_{z}$ the restriction of ad $v$ to $\mathcal{T}_{X} \otimes k(z)$; this is invertible. We let $P_{i}$ denote the $i$ th conjugation-invariant polynomial on matrices (of unspecified size). Finally let $\int_{X}: \mathrm{H}_{\mathrm{DR}}^{2 \mathrm{n}}(\mathrm{X} / \mathrm{k}) \rightarrow \mathrm{k}$ be a canonical map (cap product with the fundamental class).

Theorem 0.1. (Bott Residue Formula).

$$
\int_{X} Q\left(c_{j}\left(\mathcal{E}_{i}\right)\right)=\sum_{v(z)=0} Q\left(P_{j}\left(\left.\Lambda_{i}\right|_{z}\right)\right) \cdot \operatorname{det}\left(\left.\operatorname{ad} v\right|_{z}\right)^{-1}
$$

In Section 1 we discuss Beilinson's adeles and the sheaves $\mathcal{A}_{X}^{p, q}, \tilde{\mathcal{A}}_{X}^{p, q}$. These are analogues of the sheaves of smooth $(p, q)$-forms on a complex manifold. In Section 2 we define connections on the adelic sections $\tilde{\mathcal{A}}_{X}^{0}(\mathcal{E})$ of a vector bundle. Finally in Section 3 we prove

1991 Mathematics Subject Classification: 14F40.

Supported by an Alon Fellowship, and incumbent of the Anna and Maurice Boukstein Career Development Chair.

The paper is in final form and no version of it will be published elsewhere. 
Theorem 0.1. The proof is almost identical to Bott's proof in [Bo2]. In particular we use a projector $\omega \in \tilde{\mathcal{A}}_{X}^{1,0}$ to localize the integral to the zero locus of $v$.

I should mention other proofs of Bott's formula. Atiyah-Bott $[\mathrm{AB}]$ use a mix of analysis and topology. Carrel-Lieberman [CL] state their proof for complex manifolds, but it applies also to the purely algebraic setup.

Acknowledgements. The author thanks R. Hübl (the coauthor of [HY]), S. Kleiman who suggested the problem, and V. Hinich for explaining the Thom-Sullivan construction.

1. Adeles. Let $k$ be field of characteristic 0 , and let $X$ be a smooth $n$-dimensional projective variety over $k$. According to Beilinson, to each quasi-coherent $\mathcal{O}_{X}$-module $\mathcal{M}$ there is associated a cosimplicial sheaf $\mathbb{A}^{\cdot}(\mathcal{M})$ on $X$, the sheaf of adeles (see $[\mathrm{Be}]$ and $[\mathrm{Hr}])$. The definition of $\mathbb{A}^{q}(U, \mathcal{M})=\Gamma\left(U, \mathbb{A}^{q}(\mathcal{M})\right)$ is by a zig-zag process of direct and inverse limits, generalizing the classical adeles. (If $X$ is a smooth curve then the classical ring of adeles $\mathbb{A}(X)$ is just $\mathbb{A}_{\text {red }}^{1}\left(X, \mathcal{O}_{X}\right)$.) One has a natural isomorphism $\underline{\mathbb{A}}^{q}(\mathcal{M}) \cong$ $\underline{\mathbb{A}}^{q}\left(\mathcal{O}_{X}\right) \otimes_{\mathcal{O}_{X}} \mathcal{M}$. Denote by $\underline{\mathbb{A}}_{\text {red }}^{.}(\mathcal{M})$ the standard normalization of $\underline{\mathbb{A}}^{\circ}(\mathcal{M})$ (namely the common kernel of the codegeneracy maps), which is a complex of sheaves with coboundary operator $\partial$. Then each $\underline{\mathbb{A}}_{\text {red }}^{q}(\mathcal{M})$ is a flasque sheaf, and the natural map $\mathcal{M} \rightarrow \underline{\mathbb{A}}_{\text {red }}(\mathcal{M})$ is a quasi-isomorphism.

The adeles $\mathbb{A}_{\text {red }}^{q}(\mathcal{M})$ are a subsheaf of the product of the local factors $\prod_{\xi} \mathcal{M}_{\xi}$, where $\xi=\left(x_{0}, \ldots, x_{q}\right)$ runs over the set of reduced chains of length $q$ in $X$. For $\mathcal{M}$ coherent and $q=0$ we simply have $\mathcal{M}_{(x)}=\widehat{\mathcal{M}}_{x}$, the $\mathfrak{m}_{x}$-adic completion.

Now if $D: \mathcal{M} \rightarrow \mathcal{N}$ is a differential operator between $\mathcal{O}_{X}$-modules, there is an induced operator $D: \underline{\mathbb{A}}^{\cdot}(\mathcal{M}) \rightarrow \underline{\mathbb{A}}^{*}(\mathcal{N})$, compatible with the cosimplicial structure. Applying this to the De Rham complex $\Omega_{X / k}$ we get a cosimplicial differential graded algebra (DGA)

$$
\underline{\mathbb{A}}^{\cdot}\left(\Omega_{X / k}^{\cdot}\right)=\bigcup_{q \geq 0} \bigoplus_{p \geq 0} \underline{\mathbb{A}}^{q}\left(\Omega_{X / k}^{p}\right)
$$

Definition 1.1. For $p, q \geq 0$ let $\mathcal{A}_{X}^{p, q}:=\mathbb{A}_{\text {red }}^{q}\left(\Omega_{X / k}^{p}\right)$. Then $\mathcal{A}_{X}^{\ddot{*}}$ is a double complex, with commuting operators $\mathrm{d}: \mathcal{A}_{\mathrm{X}}^{\mathrm{p}, \mathrm{q}} \rightarrow \mathcal{A}_{\mathrm{X}}^{\mathrm{p}+1, \mathrm{q}}$ and $\partial: \mathcal{A}_{X}^{p, q} \rightarrow \mathcal{A}_{X}^{p, q+1}$, called the De Rhamadele double complex. Set $\mathrm{D}^{\prime}:=\mathrm{d}, \mathrm{D}^{\prime \prime}:=(-1)^{\mathrm{p}} \partial, \mathrm{D}:=\mathrm{D}^{\prime}+\mathrm{D}^{\prime \prime}$ and $\mathcal{A}_{X}^{i}:=\bigoplus_{p+q=i} \mathcal{A}_{X}^{p, q}$. Then $\mathcal{A}_{X}$, with Alexander-Whitney product and the operator D, is a sheaf of DGAs on $X$.

Proposition 1.2. The natural DGA map $\Omega_{X / k} \rightarrow \mathcal{A}_{X}$ is a quasi-isomorphism.

The proposition implies that $\mathrm{H}_{\mathrm{DR}}(\mathrm{X} / \mathrm{k})$ with its cup product can be calculated as $\mathrm{H}^{\cdot} \Gamma\left(\mathrm{X}, \mathcal{A}_{\mathrm{X}}\right)$. However, the DGA $\mathcal{A}_{X}$ is not (graded) commutative.

According to $[\mathrm{Ye}]$, for every maximal chain $\xi=\left(x_{0}, \ldots, x_{n}\right)$ in $X$ there is a residue map $\operatorname{Res}_{\xi}: \Omega_{X / k, \xi}^{n} \rightarrow k$. This induces

$$
\int_{X}=\sum_{\xi} \operatorname{Res}_{\xi}: \mathrm{H}^{2 \mathrm{n}} \Gamma\left(\mathrm{X}, \mathcal{A}_{\mathrm{X}}\right) \rightarrow \mathrm{k} .
$$

$\int_{X}$ coincides with cap product with the fundamental class of $X$. Thus for $k=\mathbb{C}$ we get the usual integral (up to a factor of $2 \pi \sqrt{-1}$ ).

For $\ell \geq 0$ let

$$
\Delta^{\ell}:=\operatorname{Spec} \mathbb{Q}\left[t_{0}, \ldots, t_{\ell}\right] /\left(t_{0}+\cdots+t_{\ell}-1\right)
$$


be the standard rational $\ell$-simplex, and let $\Omega^{\cdot}\left(\Delta^{\ell}\right)$ be the De Rham complex on it, which is a DGA over $\mathbb{Q}$ generated by $t_{0}, \ldots, t_{\ell}$. Then $\Omega^{\cdot}\left(\Delta^{*}\right)=\bigcup_{\ell \geq 0} \Omega^{\cdot}\left(\Delta^{\ell}\right)$ is a simplicial DGA. The definition below is extracted from [HS].

DeFINITION 1.3. Let

$$
\tilde{\mathcal{A}}_{X}^{p, q} \subset \prod_{\ell=0}^{\infty}\left(\underline{\mathbb{A}}^{\ell}\left(\Omega_{X / k}^{p}\right) \otimes_{\mathbb{Q}} \Omega^{q}\left(\Delta^{\ell}\right)\right)
$$

be the subsheaf consisting of all sections $u=\left(u_{0}, \ldots, u_{\ell}, \ldots\right)$ such that

$$
\begin{aligned}
\left(\partial^{i} \otimes 1\right) u_{\ell} & =\left(1 \otimes \partial_{i}\right) u_{\ell+1} \\
\left(1 \otimes s_{i}\right) u_{\ell} & =\left(s^{i} \otimes 1\right) u_{\ell+1}
\end{aligned}
$$

for $0 \leq \ell, 0 \leq i \leq \ell+1$. Here $\partial_{i}, s_{i}, \partial^{i}, s^{i}$ are the (co)simplicial operators. Set $\mathrm{D}^{\prime}:=\mathrm{d} \otimes 1$, $\mathrm{D}^{\prime \prime}:=(-1)^{\mathrm{p}} \otimes \overline{\mathrm{d}}, \mathrm{D}:=\mathrm{D}^{\prime}+\mathrm{D}^{\prime \prime}$ and $\tilde{\mathcal{A}}_{X}^{i}:=\bigoplus_{p+q=i} \tilde{\mathcal{A}}_{X}^{p, q}$. The sheaf of Thom-Sullivan adeles is the commutative DGA $\left(\tilde{\mathcal{A}}_{X}, \mathrm{D}\right)$.

Observe that for $p, q \geq 0$,

$$
\tilde{\mathcal{A}}_{X}^{p, q} \subset \prod_{\ell \geq 0} \prod_{\xi=\left(x_{0}, \ldots, x_{\ell}\right)}\left(\Omega_{X / k, \xi}^{p} \otimes_{\mathbb{Q}} \Omega^{q}\left(\Delta^{\ell}\right)\right) .
$$

Usual integration on the real $\ell$ simplex $\Delta^{\ell}(\mathbb{R})$ yields a $\mathbb{Q}$-linear map $\int_{\Delta^{\ell}}: \Omega^{*}\left(\Delta^{\ell}\right)$ $\rightarrow \mathbb{Q}$, such that $\int_{\Delta^{\ell}}\left(\mathrm{dt}_{1} \wedge \cdots \wedge \mathrm{dt}_{\ell}\right)=\frac{1}{\ell !}$. By linearity this extends to a map of sheaves $\int_{\Delta}: \tilde{\mathcal{A}}_{X}^{\cdot} \rightarrow \underline{\mathbb{A}}^{\cdot}\left(\Omega_{X / k}^{*}\right)$.

Theorem 1.4. ([HS]). $\int_{\Delta}$ sends $\tilde{\mathcal{A}}_{X}^{p, q}$ into $\mathcal{A}_{X}^{p, q}$, and commutes with the operators $\mathrm{D}^{\prime}$, $\mathrm{D}^{\prime \prime}$. Therefore $\int_{\Delta}: \tilde{\mathcal{A}}_{X}^{\cdot} \rightarrow \mathcal{A}_{X}$ is a homomorphism of $D G \Omega_{X / k}$-modules. For every open set $U \subset X$ the resulting map in cohomology $\mathrm{H}^{\cdot}\left(\mathrm{U}, \int_{\Delta}\right): \mathrm{H}^{\cdot}\left(\mathrm{U}, \tilde{\mathcal{A}}_{\mathrm{X}}\right) \rightarrow \mathrm{H}^{\cdot}\left(\mathrm{U}, \mathcal{A}_{\mathrm{X}}\right)$ is an isomorphism of graded $k$-algebras.

2. Connections over Adeles. Our construction is a fusion of ideas of Bott (in [Bo1]) and Parshin (in $[\mathrm{Pa}]$ ). Let $\mathcal{E}$ be a locally free sheaf on $X$, and set $\tilde{\mathcal{A}}_{X}^{p, q}(\mathcal{E}):=\tilde{\mathcal{A}}_{X}^{p, q} \otimes_{\mathcal{O}_{X}} \mathcal{E}$. Suppose we are given a family $\left\{\nabla_{(x)}\right\}_{x \in X}$, where

$$
\nabla_{(x)}: \mathcal{E}_{(x)} \rightarrow \Omega_{X / k,(x)}^{1} \otimes_{\mathcal{O}_{X,(x)}} \mathcal{E}_{(x)}
$$

is a connection over the $k$-algebra $\mathcal{O}_{X,(x)}$. Let $\xi=\left(x_{0}, \ldots, x_{\ell}\right)$ be a chain in $X$. For $0 \leq i \leq \ell$ consider the $i$-th covertex map $\partial_{(i)}^{(0, \ldots, \ell)}: \Omega_{X / k,\left(x_{i}\right)} \rightarrow \Omega_{X / k, \xi}$. By extension of scalars, $\nabla_{\left(x_{i}\right)}$ induces a connection

$$
\nabla_{\xi, i}: \mathcal{E}_{\xi} \rightarrow \Omega_{X / k, \xi}^{1} \otimes_{\mathcal{O}_{X, \xi}} \mathcal{E}_{\xi}
$$

over the algebra $\mathcal{O}_{X, \xi}$. Set

$$
\nabla_{\xi}:=\sum_{i=0}^{\ell} t_{i} \nabla_{\xi, i}: \mathcal{E}_{\xi} \rightarrow \Omega_{X / k, \xi}^{1} \otimes_{\mathbb{Q}} \mathcal{O}\left(\Delta^{\ell}\right) \otimes_{\mathcal{O}_{X, \xi}} \mathcal{E}_{\xi} .
$$

Proposition 2.1. Given a family of connections $\left\{\nabla_{(x)}\right\}_{x \in X}$, there is a unique connection

$$
\nabla: \tilde{\mathcal{A}}_{X}^{0}(\mathcal{E}) \rightarrow \tilde{\mathcal{A}}_{X}^{1}(\mathcal{E})
$$


over the algebra $\tilde{\mathcal{A}}_{X}^{0}$, such that under the embedding $(1.3),(\nabla u)_{\xi}=\nabla_{\xi} u$ for every local (algebraic) section $u \in \mathcal{E}$.

Definition 2.2. The curvature form associated to $\left\{\nabla_{(x)}\right\}_{x \in X}$ is

$$
R=\nabla^{2} \in \tilde{\mathcal{A}}_{X}^{2}\left(\mathcal{E} n d_{\mathcal{O}_{X}}(\mathcal{E})\right) \text {. }
$$

Given an invariant polynomial $P$, one has $\mathrm{DP}(\mathrm{R})=0$. The resulting Chern-Weil homomorphism

$$
\{\text { invariant polynomials }\} \rightarrow \mathrm{H}^{\cdot}\left(\mathrm{X}, \tilde{\mathcal{A}}_{\mathrm{X}}\right) \cong \mathrm{H}^{*}\left(\mathrm{X}, \mathcal{A}_{\mathrm{X}}^{*}\right),
$$

$P \mapsto[P(R)]$, is a homomorphism of $k$-algebras, independent of the connection $\nabla$.

Definition 2.3. The $i$-th Chern form of $\mathcal{E}$ with respect to the connection $\nabla$ is $\tilde{c}_{i}(\mathcal{E}, \nabla):=P_{i}(R) \in \Gamma\left(X, \tilde{\mathcal{A}}_{X}^{2 i}\right)$.

Theorem 2.4. The Chern classes $c_{i}(\mathcal{E})=\left[\int_{\Delta} \tilde{c}_{i}(\mathcal{E}, \nabla)\right] \in \mathrm{H}_{\mathrm{DR}}^{2 \mathrm{i}}(\mathrm{X})$ satisfy the Whitney Sum Formula and commute with pullback. The map $\operatorname{dlog}: \operatorname{Pic} X=\mathrm{H}^{1}\left(\mathrm{X}, \mathcal{O}_{\mathrm{X}}^{*}\right) \rightarrow$ $\mathrm{H}_{\mathrm{DR}}^{2}(\mathrm{X})$ sends the class of an invertible sheaf $[\mathcal{L}]$ to $c_{1}(\mathcal{L})$. Thus for $k=\mathbb{C}$ we get the usual Chern classes (up to a factor of $2 \pi \sqrt{-1}$ ).

3. Proof of the Formula. Denote by $Z$ the zero scheme of $v$, which is a finite reduced scheme. Choose an open subset $U \subset X$ containing $Z$, and sections $f_{1}, \ldots, f_{n} \in$ $\Gamma\left(U, \mathcal{O}_{X}\right)$, such that the corresponding morphism $U \rightarrow \mathbf{A}_{k}^{n}$ is unramified, and the fibre over the origin is the scheme $Z$. This is possible since $X$ is projective. Thus $\left.\mathcal{T}_{X}\right|_{U}$ is trivial, with a frame $\left(\frac{\partial}{\partial f_{1}}, \ldots, \frac{\partial}{\partial f_{n}}\right)$. Moreover, we can choose $U$ such that $\left.\mathcal{E}_{i}\right|_{U}$ are trivial, with frames $\underline{e}_{i}:\left.\mathcal{O}_{U}^{r_{i}} \stackrel{\sim}{\rightrightarrows} \mathcal{E}_{i}\right|_{U}$.

From here we continue along the lines of [Bo2], but of course we use adeles instead of smooth functions. The sheaf $\tilde{\mathcal{A}}_{X}^{p, q}$ plays the role of the sheaf of smooth $(p, q)$ forms on a complex manifold. The operator $\mathrm{D}^{\prime \prime}$ behaves like the anti-holomorphic derivative $\bar{\partial}$; specifically $\mathrm{D}^{\prime \prime} \alpha=0$ for $\alpha \in \Omega_{X / k}$.

Set $\mathcal{E}:=\bigoplus_{i=1}^{m} \mathcal{E}_{i}, r:=\sum r_{i}, \Lambda:=\sum \Lambda_{i}$. Then $\underline{e}=\left(\underline{e}_{1}, \ldots, \underline{e}_{m}\right)$ is a frame for $\left.\mathcal{E}\right|_{U}$. For each point $x \in U$ the isomorphism $\underline{e}: \mathcal{O}_{X,(x)}^{r} \stackrel{\simeq}{\rightarrow} \mathcal{E}_{(x)}$ induces a Levi-Civita connection $\nabla_{(x)}$ on $\mathcal{E}_{(x)}$. For $x \notin U$ choose an arbitrary connection $\nabla_{(x)}$. Let $R=\nabla^{2} \in \tilde{\mathcal{A}}_{X}^{2}\left(\mathcal{E} n d_{\mathcal{O}_{X}}(\mathcal{E})\right)$ be the resulting curvature form. Note that $R=\sum R_{i}$, and we can define

$$
P(R):=Q\left(P_{j}\left(R_{i}\right)\right) \in \tilde{\mathcal{A}}_{X}^{2 n} .
$$

$R$ decomposes into bi-homogeneous parts $R=R^{2,0}+R^{1,1}$. We will work with $R^{1,1}$. Since $\tilde{\mathcal{A}}_{X}^{p, q}=0$ for $p>n$, we get $P(R)=P\left(R^{1,1}\right)$.

One shows, like in $[\mathrm{Bo} 2]$, that

$$
L:=\Lambda-\iota(v) \circ \nabla \in \tilde{\mathcal{A}}_{X}^{0}\left(\mathcal{E} n d_{\mathcal{O}_{X}}(\mathcal{E})\right)
$$

satisfies

$$
\begin{aligned}
-\iota(v) R^{1,1} & =\mathrm{D}^{\prime \prime} \mathrm{L} \\
\left.L\right|_{z} & =\left.\Lambda\right|_{z} .
\end{aligned}
$$

Since $\nabla$ is algebraic on $U$, it follows that

$$
\left.\left.L\right|_{U} \in \mathcal{E} n d_{\mathcal{O}_{X}}(\mathcal{E})\right|_{U}
$$


For every point $z \in Z$ let

$$
\Xi_{z}:=\left\{\xi=\left(x_{0}, \ldots, x_{n}\right) \mid x_{n}=z\right\}
$$

This set of chains is the analogue of a small ball around $z$. Let $\Xi:=\bigcup_{z \in Z} \Xi_{z}$.

Given $\alpha=\left(\alpha_{\xi}\right) \in \mathcal{A}_{X}^{n, n}$, we say $\alpha$ is holomorphic (resp. has a simple pole) along a maximal chain $\xi=\left(x_{0}, \ldots, x_{n}\right)$ if for every $a \in \mathcal{O}_{X, x_{n}}$ (resp. $a \in \mathfrak{m}_{x_{n}}$ ) one has $\operatorname{Res}_{\xi} a \alpha_{\xi}=0$ (cf. [Ye] $\S 4.2$ ).

Denote the canonical pairing $\mathcal{T}_{X} \otimes \Omega_{X / k}^{1} \rightarrow \mathcal{O}_{X}$ by $\langle-,-\rangle$. It extends to a pairing $\tilde{\mathcal{A}}_{X}^{0}\left(\mathcal{T}_{X}\right) \otimes \tilde{\mathcal{A}}_{X}^{0}\left(\Omega_{X / k}^{1}\right) \rightarrow \tilde{\mathcal{A}}_{X}^{0}$

Lemma 3.1. There is a global section $\omega \in \tilde{\mathcal{A}}_{X}^{1,0} \cong \tilde{\mathcal{A}}_{X}^{0}\left(\Omega_{X / k}^{1}\right)$ such that:

(1) $\langle v, \omega\rangle=1$ on $X-Z$.

(2) $\int_{\Delta}\left(\mathrm{D}^{\prime \prime} \omega\right)^{\mathrm{n}}$ is holomorphic along any maximal chain $\xi \notin \Xi$.

(3) $\int_{\Delta}\left(\mathrm{D}^{\prime \prime} \omega\right)^{\mathrm{n}}$ has at most a simple pole along any $\xi \in \Xi$. Moreover, for any $z \in Z$

$$
\sum_{\xi \in \Xi_{z}} \operatorname{Res}_{\xi} \int_{\Delta}\left(\mathrm{D}^{\prime \prime} \omega\right)^{\mathrm{n}}=\operatorname{det}\left(\left.\operatorname{adv}\right|_{\mathrm{z}}\right)^{-1}
$$

The proof of the lemma is not difficult, but it is technical and we prefer to skip it. Let us just say that writing $v=\sum a_{i} \frac{\partial}{\partial f_{i}}, a_{i} \in \Gamma\left(U, \mathcal{O}_{X}\right)$, one can express $\omega$ in terms of the $a_{i}$.

Let $t$ be an indeterminate, and define

$$
\begin{aligned}
\eta:=P & \left(L+t R^{1,1}\right) \cdot \omega \cdot\left(1-t \mathrm{D}^{\prime \prime} \omega\right)^{-1} \\
\quad & =P\left(L+t R^{1,1}\right) \cdot \omega \cdot\left(1+t \mathrm{D}^{\prime \prime} \omega+\left(\mathrm{tD}^{\prime \prime} \omega\right)^{2}+\cdots\right) \in \tilde{\mathcal{A}}_{\mathrm{X}}[\mathrm{t}]
\end{aligned}
$$

(note that $\left(\mathrm{D}^{\prime \prime} \omega\right)^{\mathrm{n}+1}=0$, so this makes sense). Writing $\eta=\sum_{i} \eta_{i} t^{i}$ we see that $\eta_{i} \in$ $\tilde{\mathcal{A}}_{X}^{i+1, i}$. Just like in [Bo2], using formula (3.1) and Lemma 3.1, one shows that

$$
\mathrm{D}^{\prime \prime} \eta_{\mathrm{n}-1}+\mathrm{P}\left(\mathrm{R}^{1,1}\right)=0 \text { on } \mathrm{X}-\mathrm{Z} \text {. }
$$

Proof of Theorem 0.1. By definition $c_{j}\left(\mathcal{E}_{i}\right)=\left[\int_{\Delta} P_{j}\left(R_{i}\right)\right] \in \mathrm{H}_{\mathrm{DR}}^{2 \mathrm{j}}(\mathrm{X})$. From Theorem 1.4 we see that

$$
Q\left(c_{j}\left(\mathcal{E}_{i}\right)\right)=\left[\int_{\Delta} Q\left(P_{j}\left(R_{i}\right)\right)\right]=\left[\int_{\Delta} P(R)\right] .
$$

As observed before $P(R)=P\left(R^{1,1}\right) \in \tilde{\mathcal{A}}_{X}^{2 n}$. In view of formula (3.2) we must verify that

$$
\int_{X} \int_{\Delta} P\left(R^{1,1}\right)=\sum_{z \in Z} P\left(\left.L\right|_{z}\right) \operatorname{det}\left(\left.\operatorname{ad} v\right|_{z}\right)^{-1} \text {. }
$$

Now

$$
\int_{X} \int_{\Delta} \mathrm{D}^{\prime \prime} \eta_{\mathrm{n}-1}=\int_{\mathrm{X}} \mathrm{D}^{\prime \prime} \int_{\Delta} \eta_{\mathrm{n}-1}=0
$$

since $X$ is proper. Every maximal chain is either in $X-Z$ or in $\Xi$. Therefore, by (3.5)

$$
\int_{X} \int_{\Delta} P\left(R^{1,1}\right)=\int_{X} \int_{\Delta}\left(P\left(R^{1,1}\right)+\mathrm{D}^{\prime \prime} \eta_{\mathrm{n}-1}\right)=\sum_{\xi \in \Xi} \operatorname{Res}_{\xi} \int_{\Delta}\left(\mathrm{P}\left(\mathrm{R}^{1,1}\right)+\mathrm{D}^{\prime \prime} \eta_{\mathrm{n}-1}\right) .
$$

By construction the connection $\nabla$ is integrable on $U$ (it is a Levi-Civita connection there with respect to the algebraic frame $\underline{e})$, therefore on $U$ one has: $R=0, P\left(R^{1,1}\right)=0$ and 
$\mathrm{D}^{\prime \prime} \eta_{\mathrm{n}-1}=\mathrm{P}(\mathrm{L})\left(\mathrm{D}^{\prime \prime} \omega\right)^{\mathrm{n}}$. The map $\int_{\Delta}$ is $\mathcal{O}_{X}$-linear, and by $(3.3),\left.P(L)\right|_{U} \in \mathcal{O}_{U}$. Hence

$$
\int_{\Delta} P(L)\left(\mathrm{D}^{\prime \prime} \omega\right)^{\mathrm{n}}=\mathrm{P}(\mathrm{L}) \int_{\Delta}\left(\mathrm{D}^{\prime \prime} \omega\right)^{\mathrm{n}} \text { on } \mathrm{U}
$$

In view of Lemma 3.1 this concludes the proof.

REMARK 3.2. There are two easy extensions of Theorem 0.1.

(a) Dropping the assumption that the zeroes of $v$ are simple (cf. [HY]).

(b) Suppose $L \in \operatorname{End}_{\mathcal{O}_{X}}(\mathcal{E})$ is a semi-simple endomorphism. Then there are well defined classes $P_{j}(L) \in \mathrm{H}_{\mathrm{DR}}^{2 \mathrm{j}}(\mathrm{X} / \mathrm{k})$, given by $\left[\int_{\Delta} P_{j}(L+R)\right]$ for an appropriate connection $\nabla$. For example $c_{j}(\mathcal{E})=P_{j}\left(0_{\mathcal{E}}\right)$ (cf. [Bo2]). If $L$ and $\Lambda$ commute the residue formula is:

$$
\int_{X} P(L)=\sum_{v(z)=0} P\left(\left.(L+\Lambda)\right|_{z}\right) \cdot \operatorname{det}\left(\left.\operatorname{ad} v\right|_{z}\right)^{-1} .
$$

\section{References}

[AB] M. F. Atiyah and R. Bott, The moment map and equivariant cohomology, Topology 23 (1984), 1-28.

[Be] A. A. Beilinson, Residues and adeles, Funktsional. Anal. i Prilozhen. 14 (1980), 44-45; English transl. in Functional Anal. Appl. 14 (1980), 34-35.

[Bo1] R. Bott, Lectures on Characteristic Classes and Polarizations, Lecture Notes in Math. 279, Springer, Berlin, 1972.

[Bo2] R. Bott, A residue formula for holomorphic vector fields, J. Differential Geom. 1 (1967), 311-330.

[CL] J. B. Carrell and D. I. Lieberman, Vector fields and Chern numbers, Math. Ann. 225 (1977), 263-273

[ES] G. Ellingsrud and S. A. Strømme, Bott's formula and enumerative geometry, preprint (1994).

[HS] V. Hinich and V. Schechtman, Deformation theory and Lie algebra homology, preprint (1994).

[Hr] A. Huber, On the Parshin-Beilinson Adeles for Schemes, Abh. Math. Sem. Univ. Hamburg 61 (1991), 249-273.

[HY] R. Hübl and A. Yekutieli, Adelic Chern forms and the Bott residue formula, preprint (1994).

[Ko] M. Kontsevich, Enumeration of rational curves via torus actions, preprint (1994).

[Pa] A. N. Parshin, Chern class, adeles and L-functions, J. Reine Angew. Math. 341 (1983), 174-192.

[Ye] A. Yekutieli, An Explicit Construction of the Grothendieck Residue Complex (with an appendix by P. Sastry), Astérisque 208 (1992). 